\title{
Luoghi di transizione, 'eterotopie' e 'non-luoghi' - \\ Osservazioni sulla rappresentazione letteraria della stazione
}

\author{
Spaces of Transition, 'Heterotopias' and 'Non-Spaces' - \\ A Study on the Literary Representation of the Train Station
}

Julius GoldmanN [julius.goldmann@uni-wuerzburg.de]

Julius-Maximilians-Universität Würzburg, Germania

\section{RiAssunto}

L'articolo è dedicato all'analisi della rappresentazione della stazione ferroviaria nella "letteratura migrante" contemporanea, che ha saputo raccontare le metropoli italiane e i suoi landmarks con sguardo decentrato e spesso fortemente critico. Il percorso proposto si articola in quattro tappe e comprende due scrittori appartenenti al canone letterario nazionale, Carlo Emilio Gadda e Italo Svevo, e due scrittrici "migranti", Igiaba Scego, italiana di origini somale, e Anna Belozorovitch, proveniente dalla Russia. Attraverso un'analisi comparativa si cercherà di rispondere alle seguenti domande: come è cambiata la funzione letteraria della stazione? C’è una continuità nelle strutture semantiche e metaforiche della stazione tra la letteratura del primo Novecento e quella degli ultimi decenni? Quanto influiscono gli autori del canone sulla cosiddetta letteratura della migrazione? E quanto le nuove teorie dello spazio, tra cui quelle di Marc Augé (non-lieux) e Michel Foucault (hétérotopies)?

\section{Parole Chiave}

Letteratura migrante; Eterotopia; Non-luogo; Stazione ferroviaria; Modernità; Patria; Tradizione letteraria

\section{Abstract}

The study intends to analyse some literary representations of the train station in the contemporary literature written in Italian by immigrants. They have depicted some of the Italian cities and their landmarks in a quite critical way from a decentralised point of view. The study is structured into four parts and treats two canonical Italian writers, Carlo Emilio Gadda and Italo Svevo, and two "migrant" writers, Igiaba Scego, an Italian with Somali roots, and the Russian Anna Belozorovitch. Through a comparative approach, I will try to answer the following questions: How did the literary function of the station diachronically change? Is there a continuity in the semantical and metaphorical structures of the train station in the literature from the early 20th century to the present? Are there any influences on the so-called migrant writers by the canonical writers? And to what extent do the new space theories, such as the non-space (Marc Augé) and the heterotopia (Michel Foucault), affect the migrant literature?

\section{KEYWORDS}

Migration literature; Heterotopia; Non-space; Train station; Modernity; Home; Literary Tradition 


\section{Introduzione}

Fin dagli esordi della loro rappresentazione letteraria, il treno, la ferrovia e la stazione sono da considerarsi un simbolo di dinamismo, progresso e velocità. Si tratta di luoghi altamente emblematici nei quali si è condensata a livello di immaginario letterario quella che Remo Ceserani ha efficacemente definito "l'esperienza lacerante della modernità" (1993: 13); luoghi liminali capaci di realizzare una sorta di globalizzazione ante litteram. In virtù della sua modernità, la stazione è certamente uno degli spazi più rappresentativi delle metropoli europee tra Ottocento e primo Novecento. Per questa ragione, entra rapidamente nell'immaginario letterario (in testimonianze, racconti, poesie e romanzi) e viene subito rappresentata da molti autori coevi, come per esempio nel famoso romanzo La Bête humaine di Emile Zola (1890), significativo perché l'immaginario ferroviario non vi appare come un semplice contorno all'azione, ma struttura in maniera decisiva la vicenda che "fa perno sulle due stazioni terminali" (Ceserani 1993: 113) di Le Havre e la Gare Saint-Lazare di Parigi. Lo sviluppo tecnico e scientifico che ha investito l'Europa nel corso dell'Ottocento e del Primo Novecento condusse a una diffusa esaltazione del progresso, fortemente estetizzato in particolar modo dall'avanguardia futurista. Treni e stazioni come simboli di progresso, modernità e dinamismo sono esplicitamente nominati e ampiamente celebrati nel Manifesto del futurismo: "Noi canteremo le grandi folle agitate dal lavoro, [...] le stazioni ingorde, divoratrici di serpi che fumano; [...] e le locomotive dall'ampio petto, che scalpitano sulle rotaie, come enormi cavalli d'acciaio imbrigliati di tubi [...]" (Marinetti 1909). Com’è noto, tuttavia, la strada ferrata, i treni e le stazioni suscitarono reazioni contrastanti negli scrittori fin de siècle: c'era chi esaltava entusiasticamente l'innovazione e la tecnica, le nuove esperienze percettive sperimentate dal treno, la rapidità dei viaggi, e chi, invece, ne sottolineava il carattere perturbante e minaccioso, vagheggiando la bellezza della natura sventrata e distrutta dalle linee rette delle ferrovie.

Esiste un certo numero di ricerche tematiche riservate esclusivamente ai treni e alle stazioni ferroviarie nella letteratura italiana ${ }^{1}$, la maggior parte delle quali prende di mira le opere a cavallo tra Ottocento e Novecento, che è infatti l'arco temporale più significativo, perché in questo momento, anche in Italia, con un certo ritardo rispetto ad altri paesi europei, penetra e si diffonde la strada ferrata, novità prontamente registrata dalla letteratura e di fronte alla quale gli scrittori hanno reagito differentemente, con un misto di curiosità e ostilità, di entusiasmo e paura.

Una delle più interessanti risemantizzazioni della stazione nella letteratura contemporanea si deve proprio ai cosiddetti scrittori migranti che hanno riaperto i riflettori sulle "nostre" città, riraccontando le metropoli italiane ed europee con uno sguardo decentrato e spesso fortemente critico.

Riguardo al modo in cui la tradizione letteraria occidentale ha trattato il tema del treno e della stazione, mi sembra interessante indagare fino a che punto la "letteratura migrante italofona" ${ }^{\text {si }}$

1 Cfr. tra gli altri, l'imprescindibile studio di Ceserani, Treni di carta (1993), l'antologia intitolata Strade ferrate curata da Pellini et al. (1995) e le voci Treno e Stazione nel Dizionario dei temi letterari (2007) curato da Ceserani, Domenichelli e Fasano.

2 La letteratura prodotta dagli immigrati in Italia o dalle cosiddette seconde generazioni è stata variamente definita: migrante, italofona, minore, postcoloniale, multiculturale, transculturale, ecc. Sui problemi di definizione e sulle strate- 
serva degli stessi topoi o strutture narrative e fino a che punto invece introduca delle novità sul piano dei contenuti e delle modalità di rappresentazione. Nella letteratura della migrazione, sia autobiografica sia di finzione, la stazione occupa in effetti uno spazio privilegiato: essa rappresenta non soltanto il primo contatto dell'immigrato con la città, ma è anche un luogo dove gli stranieri si incontrano, scambiano merci più o meno legalmente, si riposano, si nascondono ecc. Quali sono le strategie letterarie impiegate da questi scrittori e scrittrici per descrivere le stazioni? E quali i motivi ricorrenti? In che misura è cambiata la funzione letteraria dei treni e della stazione? Quanto influiscono gli autori del canone sugli scrittori della migrazione? Questi ultimi tendono ad aderire ai modelli italiani oppure desiderano (e riescono ad) affermare una differenza?

Per rispondere a queste domande, propongo un percorso in quattro tappe che comprende due scrittori appartenenti al canone letterario nazionale, Carlo Emilio Gadda e Italo Svevo ${ }^{3}-$ qui scelti non soltanto per l'efficace rappresentazione della stazione e del treno, ma anche perché entrambi, nel tematizzare questo aspetto della modernità, hanno fatto importanti benché rapidi accenni alla questione dell'emigrazione italiana - e due scrittrici migranti, Igiaba Scego ${ }^{4}$ e Anna Belozorovitch ${ }^{5}$.

\section{Milano Centrale di Carlo Emilio Gadda}

Gadda, nel romanzo La meccanica, scritto probabilmente tra il 1928 e il 1929 e rimasto incompiuto nonostante i rimaneggiamenti successivi da parte dell'autore ${ }^{6}$, descrive la città di Milano e alcuni dei suoi landmarks (tra cui un posto privilegiato occupa la stazione) nel momento in cui l'Italia entra nella prima guerra mondiale. L'emigrazione che aveva investito massicciamente la popolazione italiana tra la fine dell'Ottocento e l'inizio del Novecento, per quanto non interrotta del tutto a causa della situazione bellica europea, subisce un momento di arresto durante il conflitto, per poi riprendere massicciamente in seguito ${ }^{7}$. Per i numerosi emigranti che durante il transito restavano bloccati alla Stazione di Milano, fu costruita, già nel 1906, una Casa degli Emigranti che Gadda descrive minuziosamente e con grande partecipazione.

Gadda introduce la sua descrizione della stazione milanese estremamente affollata in maniera classica con la presentazione complessiva dell'edificio: "Quella maladetta stazione [...]

gie di legittimazione che le diverse etichette comportano, cfr. Mengozzi (2013), in particolare la seconda parte intitolata Teorie (pp. 33-95).

3 Per la rappresentazione della stazione di questi due autori si veda lo studio di Santurbano (2009), che si occupa tuttavia di testi diversi rispetto a quelli presi in considerazione nel presente studio.

4 A voler essere precisi, Igiaba Scego non può essere definita una "scrittrice migrante" perché è nata in Italia a Roma da genitori somali. Appartiene dunque alla cosiddetta seconda generazione. La letteratura dei migranti e delle seconde generazioni presenta tuttavia molti aspetti comuni. Igiaba Scego ha pubblicato quattro romanzi e numerosi racconti che hanno richiamato l'attenzione della critica e del pubblico, tra cui Rhoda (2004) e Oltre Babilonia (2008).

5 Anna Belozorovitch è nata a Mosca nel 1983. Risiede stabilmente in Italia dal 2004. Nonostante la giovane età, è autrice di numerose opere pubblicate per la casa editrice Besa (specializzata in scritture migranti) o sulla rivista El-ghibli (rivista di letteratura della migrazione)

6 Com’è noto alcuni brani furono pubblicati sulla rivista Solaria nel 1932, mentre l'opera, o per meglio dire i capitoli ritenuti compiuti, fu pubblicata per la prima volta soltanto nel 1970.

7 Sulla storia dell'emigrazione italiana, cfr. Pugliese (2002); Bevilacqua, De Clementi, Franzina (2009). 
è gretta, meschina, antistatuaria, satura di tutte le imprevidenze che un ingegner specializzato può accumulare in un progetto d'esecuzione, con banchine larghe un metro e mezzo dove fa bisogno quaranta [...]” (1989: 509). L'elaborato sarcasmo ${ }^{8}$ di Gadda smaschera gli ovvi difetti di costruzione dell'edificio. Gadda descrive la stazione come brutta e non funzionale. La strettezza funge da premessa per aumentare il dinamismo letterario della sua descrizione:

[...] con gomitate e spigoli nelle costole e villana frequenza di tutti i villanoni del mondo universo, $[\ldots]$ quella divenne una tregenda d'inferociti e di disperati, in agguato de' convogli, ancora durante [sic!] manovra da un binario all'altro, con tutta la pena e l'ingombro de lor pacchi, bottiglie vuote, parallelepipedi in sulle spalle stracche e fagotti sferoidi in procinto di sfasciarsi [...]. (Gadda 1989: 510)

Secondo un topos ormai consolidato, l'io narrante si trova in mezzo alla folla, costretto a subire urti e spintoni. La descrizione si dissolve in immagini fortemente icastiche, dai tratti pressoché futuristi. Se, per esempio, gli zaini sono ridotti a semplici forme geometriche ("parallelepipedi”) che, nell'aprirsi, rischiano di moltiplicare il caos preesistente, gli esseri umani si inferociscono in maniera espressionistica, subendo una sorta di metamorfosi animalesca ("in agguato"), come se si stessero per preparare ad attaccare, nel giro di pochi istanti, il prossimo treno. La situazione è percepita dall'io narrante con una pletora di impressioni sinistre lievemente contrastate dalla compassione dell'io per le madri disperate: "[...] donne scarmigliate e nasali con bimbi ignudi, neri, a fargli far piscia e cacca dentro i binari, urlando i manovratori neri ed unti per salvare la nidiata dal treno imminente" (Gadda 1989: 510). La descrizione cerca di ricreare un ambiente decisivamente negativo, come è dimostrato dalla predominanza del colore nero che disumanizza sia i bambini sia i manovratori sui binari, entrambi ridotti a meri oggetti di intralcio al libero passaggio dei treni.

Il treno, però, è anche estetizzato in modo tale da opporsi drasticamente alla miseria e alle bruttezze descritte poco prima. Il suo arrivo e ingresso nella stazione sembrano esercitare un fascino irresistibile sull'io narrante, malgrado la nota ironica e beffarda sul ritardo:

[...] direttissimi con novantotto minuti di ritardo, che arrivavano a quattro per volta con un sorgere improvviso de' fari dentro l'incendio de' perduti tramonti, incedevano poi lenti, sibilanti, esausti; superstite al pulsare della celere corsa fremevano ancora da lato la dinamo stridula e il compressore del Westinghouse; e quattro per volta scaricavano su d'una sola banchina torme di bipedi e cumuli di accatastati bauli e tutta una marmaglia ancora da tutti i Gottardi e i Sempioni. (Gadda 1989: 510)

La tecnica della moltiplicazione esponenziale scatena la dinamica: Il 'treno imminente' è immediatamente trasformato nel plurale 'direttissimi', i quali finiscono per arrivare "quattro per volta". In maniera analoga lo scrittore milanese moltiplica persino i passi delle Alpi in "Gottardi e Sempioni”. Il treno è "cantato” come un oggetto estetico: come nei dipinti del tardo Ottocento

8 Sullo stile di Gadda si vedano, tra gli altri, Manganaro (1994) e Pedullà (1997). Per una presentazione globale dell'opera gaddiana, resta fondamentale Roscioni (1969). 
di Édouard Manet, il treno - rapido, improvviso e riconoscibile dai tipici fari illuminati - giunge in stazione in corrispondenza del romantico scenario del tramonto. Ma questa nota impressionistica si fonde ai peculiari tecnicismi della scrittura gaddiana, in questo caso la dinamo che trasforma l'energia cinetica in energia elettrica. Il brano si conclude in una climax debordante: i quattro treni 'scaricano' la loro merce: oggetti e persone indistintamente ("torme" e "cumuli") in una confusione spersonalizzante. L'individuo finisce per sparire ed essere assorbito nel vortice della tecnica e nel caos della dinamica degenerata. Anche questo è un motivo molto diffuso nella letteratura del primo Novecento. Gabriele D’Annunzio, per fare un esempio celebre, si serve dei medesimi motivi nel suo Trionfo della morte: "[...] nella stazione di Casalbordino, i treni versavano a brevi intervalli immense onde di popolo. [...] Si precipitavano con violenza dalle vetture; si accalcavano alla porta, contro i cancelli; urlavano e gesticolavano, respingendosi a vicenda [...]" (in Pellini et al. 1995: 47).

La rappresentazione del treno e della stazione ferroviaria presentata da Gadda ne La meccanica può dirsi esemplare: essa infatti magistralmente compendia e amalgama i topoi prevalenti della letteratura tardo ottocentesca e primo novecentesca sul tema: da un lato la stazione è un luogo impersonale, sporco, ostile all'uomo (e in particolare agli emigranti costretti a fuggire dal loro paese), dall'altro la tecnica, sulla scorta dei futuristi italiani e di altri esempi europei prima di loro, si presta a un processo di estetizzazione cui nemmeno lo scrittore milanese sembra interamente sottrarsi.

\section{Milano Centrale di Italo Svevo}

Nell'ultimo anno della sua vita (1928), Italo Svevo iniziò a scrivere la novella Corto viaggio sentimentale ${ }^{9}$. Rimasto incompiuto ${ }^{10}$ e pubblicato postumo soltanto nel 1949, il racconto "rappresenta probabilmente l'unico tentativo di romanzo ferroviario nel periodo in questione" (Santurbano, 2009: 3). Aghios, il protagonista del racconto - una tipica figura di inetto comparabile al Mattia Pascal pirandelliano - intende cambiare la sua vita e fuggire dall'ambiente alto-borghese della città meneghina. La narrazione di Svevo segue da vicino lo svolgimento dei pensieri di Aghios, il quale si appresta a compiere un viaggio di lavoro da Milano a Trieste da lui inteso soprattutto, malgrado il timore e l'esitazione, come ricerca di libertà e avventura:

Aveva vissuto troppo tempo in famiglia per poter intendere la propria passata grandezza. La famiglia era come un velo dietro al quale ci si riparava per vivere sicuri e dimentichi di tutto. [...] Come i moribondi che [...] non conoscono altro sforzo che di trattenere la vita che vuol staccarsi da loro [...]. Mancava quasi un quarto d'ora alla partenza e il signor Aghios rallentò il

9 Per una rappresentazione globale dellopera di Svevo si veda Sechi (2009). Per un approfondimento sul racconto in questione, cfr. Palumbo (2014).

10 Il racconto termina nel mezzo di una frase, anzi di una parola: "Alla stazione di Tries[te]”. Sull'incompiutezza della novella di Svevo, però, le opinioni dei critici sono divergenti. Come sottolinea Clotilde Bertoni, la curatrice dei racconti di Svevo nell'edizione delle opere diretta da Mario Lavagetto per i Meridiani, è necessario entrare nell'officina dell'autore e consultare gli autografi per evitare di incappare in errori ingenui. La stesura del racconto fu travagliatissima: tutto lascia credere che l'autore abbia scritto una versione completa poi rimaneggiata più volte. È anche probabile, come per altri racconti, che non ci siano giunte tutte le carte. 
passo. Forse aveva dimostrata troppa fretta di staccarsi dalla moglie e gli doleva ch'essa avrebbe potuto risentirsene perché, certo, essa meritava tutto, anche riguardi. (Svevo 2004: 506-507)

Il protagonista insomma vede nella famiglia un potente impedimento alla sua liberazione e per questo sogna di potersi allontanare dalla sua vita borghese perfettamente in regola attraverso questo viaggio. Significativamente la fuga in treno sembra essere la soluzione più adeguata, come già anticipato letterariamente nel Mattia Pascal di Pirandello (1904).

Analogamente allo scrittore siciliano e in sintonia tematica con la descrizione gaddiana, la stazione si presenta caotica e mal organizzata. Questo sovraffatica il protagonista di Svevo, abituato alla sua comoda vita, passata tranquillamente in famiglia. Imbranato e stordito dalla confusione, non riesce nemmeno a trovare il binario giusto:

Un treno non è una cosa piccola, ma il signor Aghios nella vasta stazione non trovava il suo. Doveva pur esserci nella stazione, in qualche posto, l'indicazione necessaria per trovarlo, ma il signor Aghios non la vedeva. Di solito sua moglie lo dirigeva. Il signor Aghios fiutò inutilmente a destra e a sinistra. Vide un facchino che gli correva incontro. Era il fatto suo. (Svevo 2004: 508-509)

Il facchino gli farà da guida attraverso la stazione che non è soltanto labirintica, mal strutturata e non funzionale ("Bisognò scendere per uno scalone sotto terra e risalire, dopo aver percorso un corridoio, alla banchina sulla quale bisognava aspettare il treno non ancora giunto da Torino" (Svevo 2004: 510)), ma anche affollata di gente, in maniera analoga al passaggio gaddiano sopra citato: "Molta gente aspettava sulla banchina. Accanto ad una colonna erano accatastati molti poveri bagagli, una sola valigia chiusa, due ceste legate, di cui una chiusa da un panno rosso e l'altra verde sbiadito" (Svevo 2004: 510). Ritroviamo in Svevo la stessa folla sulla banchina, lo stesso cumulo di oggetti e bagagli per la disposizione dei quali Svevo si serve addirittura della stessa parola di Gadda "accatastati”. Alla maniera di un pittore naturalista, Svevo descrive una montagna di bagagli, appoggiati a una colonna, semiaperti, inadeguati per un lungo viaggio in treno. Con uno zoom, lo sguardo si sofferma sui bagagli di una madre con due giovani bambini. Anche Gadda si era soffermato sulle madri disperate e timorose per i propri bambini sui binari, ma la divergenza sta nel carattere meno esagerato ed espressionistico della descrizione sveviana, nonché nella profonda compassione nei confronti di questi personaggi fugacemente incontrati dal protagonista: "Una donna sedeva sulla valigia con un poppante in grembo e una fanciullina di dieci anni, ben difesa dal freddo da un vestitino consunto, dormiva su una cesta, la testa appoggiata sul fianco della madre" (Svevo 2004: 510). Mentre Aghios si chiede se si tratti di un trasloco, la descrizione - fino a questo momento statica - viene animata dall'arrivo dal padre. Aghios intuisce il sollievo dei viaggiatori, ipotizzando che si siano potuti almeno pagare un biglietto per partire definitivamente:

\footnotetext{
"Sloggiano?" pensò il signor Aghios. Vide poi avvicinarsi un contadino che, mentre correva, esaminava dei biglietti ferroviari certo allora acquistati. La giovine donna ebbe un respiro vedendolo. Doveva aver sofferto di essere rimasta sola tanto a lungo. Quello non era un viaggio con
} 
tutta quella famiglia. Un’emigrazione, una fuga. Poi il signor Aghios non guardò più la gente che lo circondava [...]. (Svevo 2004: 510)

Aghios sembra rifiutarsi di contemplare la miseria e la realtà sociale dei suoi connazionali in quanto dirige coscientemente il suo sguardo sulle creazioni di fumo prodotte da una locomotiva fuori dalla stazione. Nel primo Novecento italiano, come è dimostrato sia dall'esempio gaddiano sia da quello sveviano, la rappresentazione della stazione è occasione da un lato per approfondire (o ironizzare sui) topoi dei tempi moderni, come il dinamismo e il progresso, dall'altro per riflettere e rendere immediatamente visibile la disperata situazione economica dei bassi ceti sociali. In altre parole, il fenomeno sociale dell'emigrazione, così consistente e traumatico per l'Italia di quegli anni, diventa a pieno titolo un elemento costitutivo delle rappresentazioni letterarie della stazione.

Gadda è forse più drastico dal momento che descrive anche il destino inumano degli emigranti italiani come operai all'estero, costretti a lavorare "nel guazzo delle cloache" (Gadda 1989: 508). Svevo, invece, con più moderazione e un lessico meno espressionistico, si serve della stazione per creare un effetto di contrasto tra il suo protagonista benestante e borghese con la miseria che lo circonda. In entrambi, la stazione - ultimo luogo della propria patria che gli emigranti possono vedere - è un luogo impersonale, ostile, freddo e pericoloso e perciò in grado di illustrare, o anche semplicemente suggerire, il destino dei migranti.

\section{Roma Termini di Igiaba Scego}

Da almeno cinquant'anni, il flusso migratorio ha cambiato direzione ${ }^{11}$. L'emigrazione, saltuariamente trattata nelle pagine degli autori italiani, cede il passo all'immigrazione raccontata dai nuovi scrittori a partire dagli anni Novanta. Come trattano il tema della stazione questi autori e in particolare le seconde generazioni (cioè le scrittrici e gli scrittori che sono in stretto contatto con due culture, quella di origine dei genitori e l'italiana nella quale sono nati e cresciuti)? È già saturo di motivi convenzionali, di strutture semantiche e metaforiche appartenenti all'immaginario ferroviario ereditato dalla tradizione, oppure l'esperienza emozionale dei nuovi migranti vi fa irruzione introducendo nuove caratteristiche?

Nel suo romanzo La mia casa è dove sono, Igiaba Scego, scrittrice nata in Italia da genitori somali, ha dedicato un intero capitolo alla Stazione Termini di Roma. Il fatto che si sia laureata in letteratura presso la Sapienza di Roma e che abbia in seguito conseguito un dottorato in pedagogia all'Università di Roma Tre ci fa legittimamente pensare che Igiaba Scego conosca piuttosto bene la tradizione letteraria italiana e che i passaggi sulla stazione ferroviaria siano almeno in parte ispirati ad alcuni grandi testi del canone nazionale. La Scego antepone un'introduzione di carattere tradizionale, soprattutto informativo, al capitolo sulla Stazione Termini, che, come gli altri dello stesso libro, ha una forte impronta autobiografica. Leggiamo l'incipit:

11 Gli studiosi calcolano che nel 1973, per la prima volta in Italia, il numero degli immigrati ha superato quello degli emigrati. Per una storia dell'immigrazione con riferimenti puntuali alle politiche migratorie adottate dai governi italiani, cfr. Einaudi (2007). 
Roma Termini è la principale stazione ferroviaria dell'Urbe e la più grande d'Italia. [...] Costruita sul colle Esquilino la stazione lavora a pieno ritmo dal 1864. L’edificio che vediamo oggi (con la facciata ad andamento orizzontale per cui la stazione è famosa) fu inaugurato solo nel 1950. La gente alla stazione corre. Si corre per un treno, per un bacio, per riabbracciare un caro appena arrivato o per fuggire dopo uno scippo. (Scego 2010: 93)

Il passaggio lascia intravedere una struttura simile al passaggio del testo di Gadda analizzato precedentemente. Anche Scego propone una breve introduzione sulla posizione topografica e la storia della stazione in questione. Fornisce inoltre numerosi dettagli architettonici per poi entrare rapidamente, con una sorta di zoom, all'interno della stazione. A quel punto, la scrittura denotativa è abbandonata, mentre lo sguardo della narratrice è tutto volto a cogliere l'atmosfera che si respira in stazione, la sua "tonalità emotiva". Losservatore in mezzo alla stazione subisce le solite "gomitate" già descritte da Gadda e Svevo, qui trasformate piuttosto in "scippi". Anche il gioco etimologico contribuisce a creare uno slittamento tra denotazione e connotazione:

Ho sempre pensato che Termini significasse "meta finale" o "fine del viaggio". Mi piaceva, suonava come un messaggio dato a noi viandanti isterici, figli della modernità. Invece ho scoperto recentemente che il toponimo Termini significa tutt'altro. Deriva dalla deformazione della parola latine 'thermae'. Nelle vicinanze ci sono infatti le terme di Diocleziano e la stazione deve a loro il suo nome. (Scego 2010: 93).

La sua fantasiosa etimologia è in realtà una metafora; indica la speranza degli immigrati che arrivano a Roma augurandosi che la loro fuga o il loro lungo viaggio siano davvero "terminati". La vera etimologia (e, dunque, simbolicamente, la storia culturale e linguistica) distrugge questa speranza in quanto spiega la vera denominazione della stazione. Mentre per la narratrice la stazione era all'inizio un punto di riferimento positivo, per molta tradizione letteraria, essa corrisponde alla definizione di "non-lieu” offerta da Marc Augé: "Si un lieu peut se définir comme identitaire, relationnel et historique, un espace qui ne peut se définir ni comme identitaire, ni comme relationnel, ni comme historique définira un non-lieu” (Augé 1992: 100). Come in molta letteratura europea ottocentesca e primo novecentesca, qui la spiegazione storica e razionale dell'etimologia demitizza la stazione, riducendola semplicemente a un luogo freddo e impersonale. Questa tensione tra due approcci alla stazione contrastanti domina anche il brano seguente, dove la narratrice si attarda a descrivere l'atmosfera emanata dalla Galleria Centrale della Stazione:

Il cuore di questa stazione è la Galleria Centrale, un cuore fisico e anche un po' metafisico [...] Non è un caso infatti che nel film Good Morning Aman il protagonista [...] percorra questa galleria con il sottofondo di una musica soul che darebbe la carica anche a un mulo moribondo. [...] A Termini anche se tutto sembra difficile, anche se c’è qualcuno che soffre tremendamente (penso ai senza fissa dimora) si ha l'illusione che un treno ti porterà via da tutti i dolori. (Scego 2010: 94)

Con il gioco di parole fra il "fisico" e il "metafisico", la Scego allude forse ironicamente ai dipinti detti metafisici di Giorgio De Chirico e Alberto Savinio (piegandoli al contempo a nuovi 
sensi): quadri "moderni" che mettono in scena dei luoghi senza individui dominati da edifici e da oggetti tecnici. Se l’intertestualità rimane implicita nel testo di Igiaba Scego, molto più esplicita è invece l'“intermedialità", come è dimostrato dai riferimenti ai film che mettono in scena l'atmosfera speciale della Galleria Centrale. Segue un racconto autobiografico che permette di aggiungere al tema della stazione un nuovo elemento, sul quale ritornerò a breve. Dopo aver rappresentato la cerimonia funebre ufficiale in Piazza del Campidoglio per dei fuggitivi somali annegati nel Mediterraneo, la Scego scrive:

"Non è il posto adatto per questo funerale" cominciò a sussurrare una vocina dentro di me. [...] Ma a rigor di logica, della mia logica, quel funerale andava fatto alla Stazione Termini, in quel grande spiazzo tra la biglietteria, il negozio della Nike e la libreria Borri. Quello era il posto giusto. L'unico luogo che a Roma potevamo chiamare davvero casa. L'unico posto davvero somalo della capitale. L'unico che ci ha accolti e ci ha chiamati fratelli e sorelle. La stazione è entrata nella mia vita, come nella vita di tutti i somali della diaspora romana, immediatamente. Non si è nemmeno presentata. (Scego 2010: 99)

A mio avviso, Igiaba Scego, precisamente in questo passaggio, aggiunge ai topoi noti della stazione una nuova variante, che richiama alla mente la riflessione foucaultiana sulle eterotopie:

Ce sont en quelque sorte des contre-espaces. Ces contre-espaces, ces utopies localisées, les enfants les connaissent parfaitement. Bien sûr c'est le grenier, ou mieux encore la tente d'Indiens dressée au milieu du grenier, ou encore, c'est - le jeudi après-midi - le grand lit des parents. C'est sur ce grand lit qu’on découvre l'océan, puisqu'on peut y nager entre les couvertures; mais ce grand lit, c'est aussi le ciel, puisqu'on peut bondir sur les ressorts [...]. Ces contre-espaces, à vraidire, ce n'est pas la seule invention des enfants ; je crois, tout simplement, parce que les enfants n’inventent jamais rien [...] La société adulte a organisé elle-même, et bien avant les enfants, ses propres contre-espaces, ces utopies situées, ces lieux réels hors de tous les lieux. (Foucault 2005: 40-41)

Scego, nel passaggio citato precedentemente, crea una relazione emozionale e personale con la stazione. Qui si concentrano i sogni degli immigrati, i quali cominciano a considerarla di conseguenza come "casa propria", oppure addirittura come una patria, benché caotica, apparentemente inospitale, disumanizzante. La stazione diventa una vera e propria eterotopia in quanto schermo sul quale i migranti proiettano la patria sognata. Essa finisce quasi per corrispondere all'esempio foucaultiano della tenda degli Indiani costruita dai bambini in contrapposizione e sostituzione del mondo reale.

La giovane protagonista, analogamente ai bambini evocati da Foucault, si costruisce un mondo fittizio allo scopo di farlo diventare la sua patria. È da notare, inoltre, che la percezione della stazione come eterotopia positiva è più intensa quando la protagonista è bambina. Questa utopia realizzata si scioglie con l'adolescenza quando la protagonista scopre anche la brutalità e l'ostilità di quel luogo. La Stazione Termini - punto di incontro e di riferimento per i Somali in diaspora - riassume su di sé questi tratti contraddittori: “Allora Termini dava loro l'impressione che Mogadiscio fosse dietro l'angolo. Bastava prendere un treno e volare via lungo i binari 
di un sogno. [...] Nessuna di queste zone sapeva coccolarti o schiaffeggiarti come la Stazione Termini" (Scego 2010: 99).

La concezione della stazione come nuova patria è dunque ambigua. Il suo carattere oscilla fra un polo positivo e negativo come suggerito dall'uso insistito di dicotomie ossimoriche come le seguenti: "Poi Termini con il tempo è diventata un'altra cosa: un microcosmo di vita e morte; una galassia di affetti; un amico caro da cui non puoi prescindere, un nemico acerrimo e cattivo. Termini ti voleva bene e ti disprezzava. Termini era una speranza, ma anche l'apocalisse" (Scego 2010: 100). La relazione fra la protagonista e il luogo diventa progressivamente sempre più negativa fino a smascherare lentamente la Stazione-Casa come eterotopia ingannatrice. Le sensazioni olfattive connotano il luogo come indiscutibilmente negativo, simile per certi versi alle descrizioni razionali della Stazione di Milano nel testo gaddiano:

[...] Termini dove succedeva tutto [...] L'unica vera stella era quella stazione malridotta. Infatti negli anni Settanta e Ottanta la stazione puzzava di piscio e non erano pochi gli angoli violenti [...] la puzza di piscio ti stordiva e sentivi nelle narici tutto il peso di una sconfitta. [...] Mi inquietava la stazione da piccola. Non ci andavo volentieri. [...] Spesso frignavo. (Scego 2010: 101)

L'illusione eterotopica interverrà proprio a mitigare e mascherare questi tratti:

Noi ci tornavamo, piegandoci alla sua volontà per la semplice illusione che ci dava. Stare lì era un po' come toccare il lembo di quella madrepatria somala sempre più lontana ed estranea. Tutte quelle chiacchiere in lingua madre e quegli odori familiari erano peggio di un trip con LSD. [...] Allora forse la mia casa era la Stazione Termini. Il principio che non dovevo dimenticare. (Scego 2010: 103)

La rappresentazione della stazione Termini nel romanzo di Scego è più elaborata di quanto non appaia a prima vista. La scrittrice riesce infatti ad amalgamare i topoi classici evidenziati tramite Gadda e Svevo con l'esperienza dei migranti somali e con il proprio personale rapporto a questo luogo della capitale, arricchendo inoltre le strutture semantiche e metaforiche della stazione grazie a dei riferimenti, più o meno espliciti, al pensiero di Foucault et di Augé.

\section{Milano Centrale di Anna Belozorovitch}

Più recente del testo di Igiaba Scego è la poesia della giovane scrittrice di origine russa Anna Belozorovitch, intitolata Stazione centrale di Milano (2012), pubblicata nel 2012 sulla rivista online El-Ghibli, specializzata in letteratura della migrazione. Si tratta di un'allocuzione in cui l'io poetico si rivolge direttamente alla stazione e descrive ciò che vede e sente dal finestrino come viaggiatrice seduta in treno mentre questo arriva alla Stazione Centrale di Milano e riparte. La poesia è incorniciata dall'insistenza sulla monotonia uniforme di spazi e oggetti visibili dal treno: l'incipit ("Pali e muri, muri e pali / uguali, senza fine, ferrovie") e l'explicit ("ultimi lampi, grigio. Uguali, / senza fine, ferrovie. / Muri e pali. / Campi”) convocano gli stessi elementi con una leggera variatio nell'ultimo brevissimo verso bisillabico. "Pali e muri, muri e pali" 
Anche nella poesia di Anna Belozorovitch - che si è laureata in Italia, in mediazione linguistica e culturale - come nel testo di Igiaba Scego, possiamo ipotizzare dei riferimenti più o meno espliciti alle più recenti teorie sullo spazio. In questa poesia, la stazione è connotata in negativo, attraverso ciò che "non è" e "non rappresenta", in modo tale da evocare indirettamente i Non-lieux di Marc Augé (1992): “Tu non sei spazio, non sei mezzo, / non sei più arte: a sé stante/ amante di casualità altrui" (El-Ghibli 2012). A differenza della posizione assunta da Igiaba Scego, la stazione non sembra avere la capacità di diventare un'eterotopia. La personificazione della stazione è caratterizzata come un'impersonale e fatalistica 'amante di casualità. Non è neanche luogo di incrocio e di incontro, ma è innanzitutto e principalmente un non-luogo, così definito dall'antropologo francese:

Le lieu et le non-lieu sont plutôt des polarités fuyantes : le premier n'est jamais complètement effacé et le second ne s'accomplit jamais totalement - palimpsestes où se réinscrit sans cesse le jeu brouillé de l'identité et de la relation. Les non-lieux pourtant sont la mesure de l'époque ; mesure quantifiable et que l'on pourrait prendre en additionnant $[\ldots]$ les voies aériennes, ferroviaires, autoroutières [...], les aéroports, les gares [...]. (Augé 1992: 101-102)

Per quanto la Belozorovitch non voglia più riferirsi in maniera esclusiva ai topoi classici della stazione, preferendo connotarla come non-luogo alla Augé, non mancano nella sua poesia né gli impliciti riferimenti alla tradizione ("sporca di sbagli e vita umana"), né le personificazioni volte a umanizzare questo spazio che diventa addirittura luogo materno ("eccoti bella / [...] / madre di strade e di speranza"), metafora già presente nel testo di Igiaba Scego, la quale si riferisce alla Stazione Termini come il luogo dove è "seppellito il [suo] cordone ombelicale" (Scego 2010: 103). Entrambe le scrittrici sembrano oscillare fra due diversi concetti, da un lato la stazione come eterotopia positiva (luogo materno e accogliente) e dall'altro come non-luogo (sporco e impersonale). Questa ambivalenza, a sua volta, pare esemplificare il modello proposto da Augé che, a ben guardare, definisce il non-luogo stesso come oscillazione delle referenze: "le premier n'est jamais complètement effacé et le second ne s'accomplit jamais totalement”. La percezione positiva e quella negativa si sovrappongono o, meglio, la seconda, in un istante, sembra scalzare la prima. Nella poesia di Anna Belozorovitch la speranza si tramuta in agonia e sofferenza: "Poi un sospiro, e l'agonia/ che soffre dentro. Si riparte. / E il rombo copre la tua voce. / La passerella slitta via" (El-Ghibli 2012). La struttura retorica posiziona i caratteri emblematici dei non-luoghi alla fine del testo. Così il concetto di eterotopia di Michel Foucault - integrato nella più recente teoria di Marc Augé - cede, in un'agonia senza emozioni, alla desolante e fatalistica immagine del non-luogo.

In conclusione, nei testi di queste due scrittrici, in maniera più o meno consapevole, sembrano combinarsi efficacemente la tradizione letteraria italiana ed europea che fa della stazione un tema centrale a cavallo tra Ottocento e Novecento con alcune moderne teorie dello spazio. Queste ultime sono usate come una sorta di attrezzo funzionale a esprimere la loro ambigua prospettiva su questi luoghi emblematici sui quali proiettano sentimenti contraddittori e che segnano il legame tra la patria (propria o dei genitori) e la "nuova" terra, percepita talvolta come ostile talaltra come lo scenario di una ritrovata libertà. 


\section{Riferimenti bibliografici}

Augé, M. (1992). Non-lieux. Introduction à une anthropologie de la surmodernité. Paris: Seuil.

Belozorovitch, A. (2012). Stazione centrale di Milano. El-Ghibli [online], 37. In: http://archivio.el-ghibli. org/index.php\%3Fid=1\&issue=09_37\&section=1\&index_pos=2.html.

Bevilacqua, P., De Clementi, A., \& Franzina, E. (Eds.) (2009). Storia dellemigrazione italiana. 2 Vols. Roma: Donzelli.

Ceserani, R., Domenichelli, M., \& Fasano, P. (Eds.) (2007). Dizionario dei temi letterari. Torino: UTET.

Ceserani, R. (1993). Treni di carta. L'immaginario in ferrovia. L'irruzione del treno nella letteratura moderna. Genova: Marietti.

Einaudi, L. (2007). Le politiche dellimmigrazione in Italia dall'Unità a oggi. Bari: Laterza.

Foucault, M. (2005). Die Heterotopien. Der utopische Körper. Frankfurt am Main: Suhrkamp.

Gadda, C. E. (1989). La meccanica. In: G. Pinotti, D. Isella, \& R. Rodondi (Eds.), Romanzi e racconti II (pp. 461-590). Milano: Garzanti.

Manganaro, J.-P. (1994). Le Baroque et l'Ingénieur. Paris: Seuil.

Marinetti, F. T. (1909). Fondazione e manifesto del futurismo. Milano: Direzione del Movimento futurista.

Mengozzi, C. (2013). Narrazioni contese. Vent'anni di scritture italiane della migrazione. Roma: Carocci.

Palumbo, M. (2014). La meta e il viaggio. Osservazioni su Corto viaggio sentimentale di Italo Svevo. Italies [online], 17-18. In: http://italies.revues.org/4917\#quotation.

Pellini, P., Polacco, M., \& Zanotti, P. (Eds.) (1995). Strade ferrate. La tematica del treno e della ferrovia nei testi di Jules Verne, Gabriele d’Annunzio, Gabriel García Márquez e parecchi altri scrittori. Pisa: Nistri-Lischi.

Pedullà, W. (1997). Carlo Emilio Gadda. Il narratore come delinquente. Milano: Rizzoli.

Pugliese, E. (2002). L'Italia tra migrazioni internazionali e migrazioni interne. Bologna: Il Mulino.

Roscioni, G. C. (1995). La disarmonia prestabilita: studio su Gadda. $3^{a}$ ed. Torino: Einaudi.

Scego, I. (2004). Rhoda. Roma: Sinnos.

_. (2008). Oltre Babilonia. Roma: Donzelli.

- (2010). La mia casa è dove sono. Milano: Rizzoli.

Santurbano, A. (2009). Viaggi di carta e modernità: la presenza della ferrovia nella narrativa italiana del primo Novecento. Annali della Facoltà di Lettere e Filosofia, XLIV, 183-195.

Sechi, M. (Ed.). (2009). Italo Svevo. Il sogno e la vita vera. Roma: Donzelli.

Stierle, K. (1993). Der Mythos von Paris. Zeichen und Bewusstsein der Stadt. München: C. Hanser.

Svevo, I. (2004). Racconti e scritti autobiografici. Milano: Mondadori. 